\title{
POCC Regimen
}

National Cancer Institute

\section{Source}

National Cancer Institute. POCC Regimen. NCI Thesaurus. Code C9629.

A chemotherapy regimen consisting of procarbazine, vincristine, cyclophosphamide, and lomustine (CCNU) that may be used in the treatment of melanoma. 